\section{Zur historischen Authentizität ägyptischer und biblischer Quellen: Der Palästinafeldzug Schoschenks I. ${ }^{1}$}

\section{Karl Jansen-Winkeln}

Der Autor dieser an der John Hopkins University eingereichten Dissertation ist Alttestamentler und im "Nebenfach“ Ägyptologe. Daher war eine erneute Behandlung des berühmten Feldzugs Schoschenks I. nach Palästina naheliegend, denn er ist sowohl durch ägyptische wie auch durch biblische Quellen bezeugt. Das wichtigste ägyptische Zeugnis ist die Triumphdarstellung auf der Außenwand des „Bubastide Gate“ beim 2. Pylon von Karnak, und tatsächlich sind die einzelnen Elemente dieser seit Champollion bekannten Darstellung zwar oft behandelt worden, vor allem die Ortsnamenliste, aber noch nie alle im Zusammenhang. Eine erneute Untersuchung von Sinn und Zweck dieser Quelle ist also keineswegs überflüssig.

$D_{\text {as }}$ erste Kapitel $(1-15)$ gibt einen Überblick über die bisherigen Studien des Feldzugs durch M. Noth, B. MaZar, S. Herrmann, W. Helck, Y. Aharoni, K. Kitchen, G. Ahlström und F. Clancy und kommt zu einem recht abgewogenen Urteil über deren Verdienste und Schwächen. Die meisten Autoren haben anhand der Ortsnamenliste versucht, den Weg zu rekonstruieren, den Schoschenks Armee genommen hatte, aber keine dieser Rekonstruktionen ist wirklich überzeugend: man muß entweder Lücken in der Route oder unübliche Lesungen in Kauf nehmen oder die Reihenfolge der Ortsnamen bzw. die Leserichtung der Zeilen ändern, um zu einem einigermaßen glaubwürdigen Verlauf des Feldzugs zu gelangen. Außerdem sieht Wilson einige wichtige Fragen durch keine der bisherigen Untersuchungen gelöst: Warum griff Schoschenk (der Ortsnamenliste zufolge) vor allem Israel an, das Gebiet seines früheren Schützlings Jeroboam? Und warum berichten umgekehrt die entsprechenden Passagen der biblischen Bücher einen Angriff gegen Jerusalem, also gegen Juda?

$\mathrm{U}_{\mathrm{m}}$ diese Fragen $\mathrm{zu}$ beantworten und $\mathrm{zu}$ einem kohärenten Bild des Feldzugs zu kommen, untersucht er im zweiten Kapitel (16-47) die wichtigsten vergleichbaren Triumphdarstellungen aus dem Neuen Reich, und zwar drei von Thutmosis III., zwei von Sethos I., zwei von Ramses II. (alle in Karnak) und zwei von Ramses III. in Medinet Habu. Seine (begrüßenswerte) Absicht

Zugleich eine Besprechung zu Wilson, Kevin A.: The Campaign of Pharaoh Shoshenq I into Palestine. Tübingen: Mohr Siebeck 2005. VIII, 151 S. m. Abb. $8^{\circ}=$ Forschungen zum Alten Testament 2. Reihe, 9. Kart. 39.00€ ISBN 3-16-148270-0. dabei ist, alle Elemente dieser Kompositionen für die Deutung zu berücksichtigen, nämlich Triumphszene mit Beischriften, Willkommensrede des Amun und Ortsnamenliste(n) samt ihren Überschriften, und nicht, wie früher häufig geschehen, nur die Ortsnamenlisten auszuwerten.

Im dritten Kapitel (48-65) folgt eine (kurz) kommentierte Übersetzung und Beschreibung der Triumphszene Schoschenks I. Die zahlreichen Ortsnamen und die Vorschläge für ihre Identifizierung werden aber nicht hier, sondern in einem längeren „Anhang“ (101-133) erörtert. Als nächstes (Kapitel 4, 66-74) bespricht der Autor die wenigen anderen ägyptischen Texte, die möglicherweise auf denselben Feldzug nach Palästina anspielen: eine Felsstele Schoschenks I. am Gebel es-Silsila, Bruchstücke einer Stele aus Karnak, ein vereinzeltes biographisches Beiwort eines thebanischen Priesters und Sohn eines Wezirs auf seiner Mumienkartonage und ein kleines Stückchen von einer in Megiddo errichteten Stele, auf dem glücklicherweise noch der Name Schoschenks I. erhalten ist.

Im fünften Kapitel (75-96) werden dann die biblischen Erwähnungen des Feldzugs (1 Kön 14,25-28; 2 Chr 12,1-12) besprochen sowie diejenigen Passagen, die in denselben historischen Zusammenhang gehören (Rebellion des Jeroboam, seine Flucht nach Ägypten etc.). Das sechste und letzte Kapitel (97-99) enthält Wilsons Beurteilung der ganzen Affäre.

Die Behandlung der ägyptischen Texte in Kapitel 3 und 4 ist nicht immer überzeugend.

$\mathrm{Zu}$ den Beischriften und Reden der Triumphszene (Kapitel 3):

p. 48/49 (a): Im Horusnamen Schoschenks I. ( $k 3$ nbt mrij $R^{\varsigma} s b^{c} . f m$ nswt $r$ zm3 t3 wj) kann $s b^{c} . f$ nicht als „who rises“ verstanden werden, auch wenn $s b^{c}$ gelegentlich intransitiv gebraucht wird. Es ist natürlich „den er [Re] erscheinen ließ ..." zu verstehen, wie auch der ganz ähnliche Horusname Osorkons II. $\left(s b^{c} s w R^{c}\right)$ zeigt.

p. $53 \mathrm{ff}$.: Für die lange Ansprache Amuns an den König gibt es eine neuere Umschrift und Übersetzung, die Wilson nicht berücksichtigt hat: K. A. Kitchen, Poetry of Ancient Egypt, Jonsered 1999, 433-40 (s. dazu auch J. Quack, Or 69, 2000, 432).

p. 53/54, Z. 5: Umschriften wie $b \underline{d}-b p r-n-r^{c}$ („HedjKheperen-Re“) dürften eigentlich nicht vorkommen.

Z. 7: In der Mitte von Z. 7 ist die Textwiedergabe des Epigraphic Survey ausnahmsweise einmal nicht ganz korrekt, wie das Foto zeigt. Es ist nicht $p s$, sondern $q(3)$ s zu lesen, s. Biblische Notizen 49, 1989, 11-12. Im Zusammenhang: „Du bist siegreich ausgezogen, du bist machtvoll heimgekehrt, nachdem du [die Beiden Länder (?)] vereinigt hattest. (Ich) habe für dich [alle (s. KRI IV, 
24,3)] Fremdländer gefesselt, die Ägypten nicht kannten und es gewagt hatten, [seine (?)] Grenzen anzugreifen."

Z. 8: Sicher nicht sndt.k sdn. n.s jbw b3swt „as for your fear, it has punished the hearts of the foreign lands", sondern sndt.k sd\{n\}.n.s jbw b3swt „die Furcht vor dir hat die Herzen der Fremdländer zerbrochen“, vgl. KRI I, 30,8; II, 160,12; V,92,15-6: hrryt.k s d. n.s b3swt "der Schrecken vor dir hat die Fremdländer zerbrochen" (dort auch jeweils die gleiche Fortsetzung: jw.k mj $\mathrm{Hr}$ $\left.b r-t p t 3 w_{j}\right)$. Die Schreibung $s d n$ statt $s d$, die Wilson irregeführt hat, ist wohl durch das folgende Formativ $n$ bedingt. Er hätte gut daran getan, die zahlreichen ramessidischen Parallelen zu einzelnen Phrasen dieser Rede ${ }^{2}$ anzuführen und in seiner Übersetzung auch zu berücksichtigen.

Z. 9: wnwn „sich bewegen“ paßt nicht zu den Spuren. Wilson hat hier ein Wortspiel verkannt (ebenso Kitchen, op. cit., 434/5), es ist wohl zu lesen: jw.k $m$ wn[t]j wn br bftjw.k tj.n.k sbjw r.k "du bist ein Triumphator, der über deinen [statt: seinen] Feinden ist, nachdem du die niedergetreten hast, die gegen dich rebellieren." Das Wort wntj (s. JEA 29, 1943, 30-1) wird hier in seinen beiden Bestandteilen wn und $t j$ wiederaufgenommen und „ausgedeutet". Das ist auch der Grund dafür, dass man das altertümliche Simplex tj (WB V, 237,10-12) statt des üblichen $t j t j$ verwendet hat.

Z. 10-17: Vgl. auch M. Ullmann, König für die Ewigkeit - Die Häuser der Millionen von Jahren, ÄUAT 51, 2002, 571.

Z. 13-14: prj.k [j]m.j r jrr nd dtj.j nicht „may you go out from me ... “, sondern „du bist aus mir hervorgegangen / aus mir entstanden, um mein Beistand zu sein“.

Z. 16 ist keineswegs unklar (vgl. p. 56 q): „Du hast begonnen, Denkmäler zu errichten im südlichen und nördlichen Heliopolis (und in) jeder Stadt desgleichen für den (jeweiligen) Gott, der in seinem Kultbereich ist."

Z. 17: Wilson liest $b r . k(w i) h r[. s]$ "how happy I am with [it]", aber das Pseudopartizip der 1. Person kann so nicht gebraucht werden; es ist sicher brw jb.k $h r$ „dein Herz sei zufrieden über" zu verstehen. Das folgende " $h$ c kann aufgrund des Determinativs nur die „Lebenszeit“ bedeuten, nicht „to stand“.

Z. 18: $q n m z p(w)[n] n b t w$ „stark in Taten des Sieges".

Z. 19: nšnj.n bh.k $m$ sd t $r$ pḥ̂w [n]w t3 w ‘ $b 3(w) r t 3$ nb $d m \underline{d}(w)$,dein Gluthauch hat gewütet bis zu den Enden der Erde (Wb I, 538,4), indem er gegen alle Länder zusammen gekämpft hat"; die Wiedergabe des Epigraphic Survey ist hier stellenweise zweifelhaft, vgl. das Foto.

Z. 21: „You made a great slaughter (jrj.n.k b3yt $3 t$ ) among them without their limit", aber "among them“ (jm.sn) steht nicht da. Es ist wohl mwtw.sn $n$ drw.sn zu verstehen „indem ihre Toten zahllos (Wb V, 588,1) waren".

2 Vgl. jetzt R. Gozzoli, The Writing of History in Ancient Egypt during the First Millenium BC (ca. 1070-180 BC). Trends and Perspectives, London 2006, 30-31 mit einer (keineswegs vollständigen) Liste dazu.
Z. 23: $3 r . j n . k$ jrjw-mšc $n w$ Mtn $n$,ich bedränge für dich die Truppen von Mitanni“, sicher nicht „as for the army of Mitanni“; zum Gebrauch des anachronistischen Namens „Mitanni“ vgl. M. Görg, GM 207, 2005, $5-6$.

\section{Zu Kapitel 4:}

Für das Stelenfragment Kairo 3/12/24/1 aus Karnak (p. 68-9) hätte Wilson unbedingt die Bearbeitung von B. Grdseloff heranziehen müssen (in: Revue de l'histoire juive en Egypte, 1, 1947, 95-7), der auch ein weiteres Fragment einbezogen hatte und gegenüber Legrain deutlich verbesserte Lesungen und Ergänzungen bietet. Aber auch davon abgesehen enthält Wilsons Text einige krasse Irrtümer:

Z. 1 [= Z.3]: $m$ di.w (mit Suffix .w) kann nicht ,in giving victory" bedeuten.

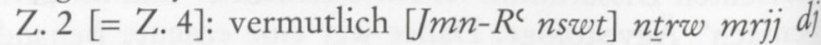
' $n b \underline{d} t$ "[König ... geliebt von Amonraso]nther, dem ewig Leben gegeben ist".

Z. 3 [= Z. 5]: b3 wtj ist sicher nicht „formost“, sondern es ist mit Grdseloff [n3j]j.j b̧3wtjw "meine Befehlshaber" zu ergänzen.

Z. 9 [= Z. 11]: Wilson liest ' $b 3 . s n n . i \operatorname{snf} n q n$ [] „they present to me the blood of brave [men]", was ganz unidiomatisch wäre. Statt dessen fängt mit dj.sn natürlich ein neuer Satz an: „dj.sn n.j jsw $m$ qn [...] „mögen sie mir den Lohn geben bestehend aus Stärke [...]

NB. Die wenigen verstümmelten Sätze dieses Fragments erlauben es nicht, eine direkte Verbindung zu dem bekannten Feldzug Schoschenks nach Palästina zu ziehen. Immerhin aber geht es um eine militärische Auseinandersetzung im Nordosten Ägyptens. Wilsons Behauptung, "there is no reason to assume that this was in any way connected with the campaign mentioned in the Bible" scheint doch etwas apodiktisch.

Die wichtigsten Ergebnisse enthält das zweite Kapitel, die vergleichende Untersuchung der "Triumphal Reliefs". Wilson kommt zu folgenden Schlüssen:

a) Sinn und Zweck der Triumphszenen (p. 36-9): Die bildliche Darstellung zeigt den König in der Regel beim Niederschlagen von Fürsten verschiedener Länder und Regionen, ebenso sind die Gefangenen unterschiedlicher Herkunft, z. B. Nubier und Asiaten. Daher handelt es sich nicht um einen Triumph nach einer. konkreten Schlacht, sondern es werden damit alle Siege des Königs gefeiert. Auch die begleitenden Inschriften beziehen sich auf Niederlagen ganz unterschiedlicher Länder oder Volksgruppen wie der Neunbogenvölker, der Beduinen, Asiaten und Nubier, oft mit allgemeinen Bezeichnungen für bestimmte Regionen. Daher dürfte auch die $\mathrm{Na}^{-}$ mensliste einem ähnlichen Zweck dienen: Die oft große Zahl der Ortsnamen soll nichts weiter ausdrücken, als dass der König "die ganze Welt" erobert hat. Die Triumphszene hat insgesamt religiöse Funktion, sie hält die Feinde Ägyptens in Schach. Die Anlage bestimmter Relieffolgen in Karnak und Medinet Habu zeigen, das ${ }^{S}$ die Bilder konkreter Siege in den Triumphszenen kulminieren, die somit eine Art Zusammenfassung und Überhöhung der königlichen Siege zum Wohle des Tempels darstellen. 
b) Die Quellen der Ortsnamenlisten (p. 40-6). Wilson diskutiert drei Möglichkeiten:

Erstens, die Ortsnamen könnten auf Eintragungen in Kriegstagebüchern o. ä. beruhen. Dann stellt sich die Frage, was die Ortsnamen eigentlich zu bedeuten haben. Die seit M. Noth bevorzugte Variante, darin eroberte Orte oder zumindest die Stationen eines Feldzugs zu sehen, lehnt Wilson ab: Die Orte seien ohne größere Umstellungen meist nicht zu einer sinnvollen Route zu verbinden. Zudem enthält eine Liste Thutmosis' III. nicht weniger als 350 Orte, die kaum alle vom Feldzug betroffen worden sein können. Und die Überschrift sagt ausdrücklich, dass diese Orte als Feinde des Königs bei Megiddo versammelt waren. Man könnte daher denken, die Ortsnamenlisten seien eine Aufzählung der Feinde, gegen die sich der Feldzug richtete. Aber auch das kann nicht richtig sein, da die Listen ja auch manche Orte enthalten, die entweder keine Feinde Ägyptens waren oder sogar Verbündete. Wilson erklärt das damit, dass die Listen nicht nur Feinde, sondern auch potentielle Feinde Ägyptens enthielten. Damit würden aber die Kriegstagebücher, die sich ja mit den realen Gegebenheiten des Tages beschäftigen sollten, eigentlich als mögliche Quellen ausscheiden.

Zweitens könnten, nach einem Gedanken D. Redfords, den Listen Reisebeschreibungen zugrundeliegen.

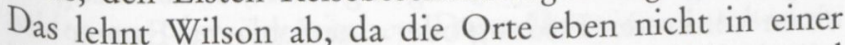
sinnvollen geographischen Reihenfolge erscheinen und außerdem derartige Texte nicht überliefert seien.

Drittens könnten die seit dem Mittleren Reich bezeugten Onomastika für die Ortsnamenslisten herangezogen worden sein. Diese Möglichkeit scheint Wilson zu favorisieren: In den Onomastika sind die Ortsnamen wie in den Listen - zum Teil in ihrer geographischen Abfolge aufgereiht, zum Teil aber auch nicht. Die Onomastika wurden, wie die Ortsnamenlisten, von Schreibern kompiliert und in den Schreiberschulen auch ständig aktualisiert. Das heißt, „topographical lists could have been compiled easily when needed. The artists who carved the triumphal reliefs would only have had to ask the scribes for a certain number of names - possibly specifying the region from which the names should be drawn - and the scribes would draw up a list. This would explain how the number of toponyms in a list was al ways the exact number needed to fill the available

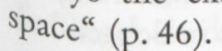

$\mathrm{Ob}$ nun Kriegstagebücher die Quelle waren oder Onomastika, Wilson zufolge können die Ortsnamenlisten keine historischen Informationen über einen bestimmten Feldzug enthalten: Im ersten Fall enthalten sie die Namen sowohl von tatsächlichen wie auch von potentiellen Feinden Ägyptens [?], ohne dass man zwischen ihnen unterscheiden könnte, im zweiten Fall enthalten sie nur Namen von Orten, die den Schreibern dieser Zeit bekannt waren, ohne Angabe darüber, ob diese Orte erobert oder passiert wurden, ob sie Feinde oder Verbündete waren. Ortsnamenlisten sind mithin für die Rekonstruktion ägyptischer Kriege oder Feldzüge wertlos (p. 46).

Diese Schlüsse gelten auch für die Interpretation der Triumphdarstellung Schoschenks I. (p. 63-65): Es wird
- wie in den anderen Triumph-Reliefs, keine spezifische Schlacht erwähnt, der König soll nur als Triumphator über alle seine Feinde erscheinen, die daher auch nur sehr allgemein benannt werden. Die Inschrift enthält keinerlei historische Informationen, ebensowenig wie die bildliche Darstellung. Daher sollte auch die Ortsnamenliste auch in diesem historisch unverbindlichen Sinne verstanden werden: sie gibt nur einen (allgemeinen) Katalog von Ortsnamen, möglicherweise einfach onomastischen Listen entnommen, der wiederum nur die Herrschaft des Königs über alle Länder dokumentieren soll.

Diese Ergebnisse scheinen mir nicht recht überzeugend.

Wilsons These, die Triumphdarstellungen seien historisch mehr oder weniger belanglos ("not intended to provide historical information about the campaigns of the king") scheint zunächst die Tatsache für sich zu haben, dass sie grundsätzlich nicht datiert sind und auch keine konkrete Schlacht nennen oder beschreiben. Auf der anderen Seite sind derartige Darstellungen ausschließlich für solche Könige bezeugt, die nachweislich Feldzüge geführt haben. Wäre es nur darum gegangen, die Tempel mit Bildern der Macht des Königs und apotropäischen Szenen zu schmücken, wieso haben nicht auch die anderen Könige, die wir als Bauherren kennen, solche Szenen anbringen lassen?

Was die Ortsnamenlisten angeht, so ist es sicher richtig, dass die Rekonstruktionen von Feldzugsrouten, für die man sie benutzt hat, kaum je wirklich überzeugend waren. Aber allein die Tatsache, dass man sie so interpretieren kann, zeigt doch, dass es sich bei ihnen nicht um einen allgemeinen „catalogue of foreign lands" handelt, der eine Auswahl der tatsächlichen oder möglichen Feinde Ägyptens oder gar alle enthält. In diesem Fall würde man doch Orte aus der gesamten Umwelt Ägyptens erwarten, auch aus der Ägäis, aus Libyen oder Kleinasien. Tatsächlich ist die Welt dieser Listen aber sehr viel begrenzter: in aller Regel bestehen sie ganz überwiegend aus Ortsnamen derjenigen Region, wo der betreffende König Krieg geführt hat, und das gilt in ganz besonderem Maße von der Schoschenk-Liste. Auch Wilsons sagt deutlich, dass sie „important data for historical geography" (p. 65) enthalte, und das tut sie ja auch, aber die Beschränkung auf Palästina paßt nicht zu der von ihm vorgeschlagenen Funktion ,to serve as a catalogue of foreign lands". Auch die zahlreichen Mehrfachnennungen derselben Ortsnamen, ${ }^{3}$ die schwerlich allesamt gleiche Namen für verschiedene Orte sein können, wären bei diesem letzteren Zweck nicht zu erwarten; sie passen allerdings auch nicht recht zu einer Liste eroberter Städte.

Sinn und Funktion eines Textes lassen sich oft seiner Überschrift entnehmen, wenn er denn eine hat, und die Ortsnamenlisten der großen Triumphszenen haben tatsächlich oft Überschriften. Wilson führt die wichtigsten darunter auf (p. 26-35), aber trotz seiner Feststellung

Vgl. B. U. Schipper, Israel und Ägypten in der Königszeit, OBO $170,1999,128$, n. 76. 
auf p. 27 (,these superscriptions indicate how the topographical lists are to be understood") macht er nur sehr begrenzten Gebrauch davon. Die Überschriften der großen Listen Thutmosis' III. mit den "Nordvölkern“ (p. 26-29, s. Urk IV, 779-81) sagen deutlich, dass die Orte darin (d.h. ihre militärischen Kontingente und/ oder ihre Herrscher) diejenigen sind, die der König in Megiddo eingeschlossen hatte und deren Kinder er gefangen nach Ägypten brachte. Nimmt man diese Überschriften ernst, ist es klar, dass man die Ortsnamen dieser Listen Thutmosis' III. nicht dazu verwenden kann, die Route seines Feldzugs zu rekonstruieren, und das geht auch aus der Lage der Orte und ihrer Anordnung hervor (p. 28-9). Soweit wird man Wilson unbedingt zustimmen. Dagegen zitiert er zwar auch mehrere der anderen Überschriften (p. 30-34), aber ohne die naheliegenden Folgerungen daraus zu ziehen.

Als Beispiel die Überschrift einer Liste Ramses' II. am Westteil der Südwand des Hypostylensaals von Karnak (KRI II, 161, 8-9): „Liste dieser südlichen und nördlichen Fremdländer, die seine Majestät geschlachtet hat, indem ein großes Massaker unter ihnen angerichtet wurde, ohne dass man die Zahl kennt, und ihre Einwohner als Gefangene weggebracht wurden, um die Speicher seines Vaters Amun-Re, des Herrschers von Theben, zu füllen, so dass alle Fremdländer (nun) Hörige seiner Majestät sind." Wörtlich gleiche oder sinngemäß ganz ähnliche Überschriften haben auch Listen von Amenophis II. (Urk IV, 1334-5), Sethos I. (KRI I, 28,14; 31,16), Ramses II. (KRI II, 163,11-12; 177,6-7) und Ramses III. (KRI V, 99,8-9): sie alle sagen expressis verbis, dass eine militärische Auseinandersetzung stattgefunden hat und Gefangene gemacht wurden, die dann dem Tempel des Amun zugewiesen worden sind. Auch das passt schlecht zu Wilsons These, dass diese Listen nur den allumfassenden Herrschaftsanspruch des ägyptischen Königs illustrieren sollen. ${ }^{4}$

Eine andere Deutung liegt näher: $\mathrm{Da}$ alle diese Triumphszenen und ihre Listen in Tempeln des Amun angebracht worden sind, hat der Hinweis auf das „Füllen der Speicher" des Amun mit Gefangenen sicher besondere Bedeutung. Zudem werden in den Listen ja keine „Orte“ dargestellt, sondern (gefesselte) Gefangene, die mit "Ortsschildern" gekennzeichnet sind. Es wäre also zu überlegen, ob die Ortsnamenlisten nicht einfach die Herkunftsorte der Gefangenen angeben, die der König auf einem Feldzug (oder auch mehreren) gemacht und dem Tempel übergeben hat. Eine solche Annahme würde gleich mehrere Schwierigkeiten erklären:

Die Orte sind in der Regel nicht in einer fortlaufenden topographischen Reihenfolge aufgeführt, so dass sich problemlos eine durchgehende "Route“ eines Feldzugs ergäbe (vgl. Wilson, p. 29; 31-32); sie sind aber auch nicht ganz ungeordnet. Vielmehr bestehen die Listen vielfach aus Gruppen benachbarter Orte, oder, wie Kitchen es ausgedrückt hat: „the rows of place-

Allenfalls die Überschrift der Liste Ramses' III. am südlichen Pylon von Medinet Habu (KRI V, 95,4-5) könnte man so verstehen, aber sie ist ein Einzelfall. names ... do not run in a continuous sequence (like an entire journey), but are made up of segments or extracts from routes. ${ }^{\text {5 }}$ Hätten die Kompilatoren dieser Listen aber wirklich beabsichtigt, die von einem Feldzug betroffenen Orte zu nennen, wären diese Aufteilung in (vielfach disparate) „Segmente" schwer erklärlich. Falls es sich aber um Herkunftsorte von Gefangenen handelt, sind derartige Gruppierungen von „Nachbarn“ leicht verständlich. Ebenso verständlich wären dann mehrfache Nennungen derselben Namen und das Vorkommen von Orten, die von dem Feldzug gar nicht betroffen waren (Wilson, p. 29; 32). Auch die erstaunliche Vielzahl der Namen in manchen Listen (Wilson, p. 42) erklärt sich dann leicht, ebenso die gelegentliche Nennung ganzer Regionen statt einzelner Orte (p. 32): das wäre dann naheliegend, wenn Gefangene „auf dem Land“ wohnten.

Im Gegensatz zu den Listen enthalten sowohl die Beischriften zur Triumphszene selbst (Erschlagen der Fein de) als auch die Rede Amuns an den König (Kitchens „Triumphal Welcoming-Speech“) meist nur generelle (b3 swt nbwt št 3 wt) und/oder altertümliche Bezeichnungen der Feinde (Jwntjw, Mntjw, t3w nbw fnbw nw bmowt Sttt etc.), und sie sind sich alle in Phraseologie und Inhalt sehr ähnlich. Die kriegerischen Aktivitäten des Pharao und die Hilfe Amuns dabei werden ausschließlich in allgemeinen Wendungen gefeiert, ohne Bezug auf einen konkreten Feldzug oder eine Schlacht. Es war also offenbar ein Dekorations- und Textmuster, das (mit jeweils geringfügigen Änderungen) zur „Wiederverwendung" geeignet war. Würden die Ortsnamenlisten, wie oft angenommen, die Etappen eines Feldzugs wiedergeben, dann wäre der Widerspruch zwischen den einzelnen Elementen der Triumphdarstellungen in der Tat erstaunlich: die Listen beschreiben einen bestimmten Feldzug, Beischriften zur Darstellung und Götterrede sprechen nur allgemein von der kriegerischen Macht des Königs. Wurden derartige Darstellungen aber anläßlich der (feierlichen) Übergabe der Gefangenen an den Tempel angebracht, ist es verständlich, dass sie in der Regel nicht auf einen Feldzug oder eine Schlacht Bezug nehmen: bei ihrer Übergabe an den Tempel wurde nur die überragende Macht dessen betont, der sie (unter welchen Umständen auch immer) gefangen genommen hatte, und die Hilfe dessen, der sie nun bekam. Daraus ergibt sich schon, dass die Triumphszenen natürlich auch religiöse Funktion haben und die Feinde Ägyptens in Schach hat ten sollen, wie Wilson betont hat. Wenn den Ortsnament listen oft die „Neunbogenvölker" vorangestellt werden, wie auch im Fall der Schoschenk-Liste, dürfte das ebent falls in diesem Sinne zu verstehen sein: die Übergabe des Gefangenen an Amun ist ein Zeichen dafür, dass der König alle Feinde unterworfen hat. Aber daraus kan man nicht schließen, dass diese Darstellungen keiner lei Bezug zur historischen Realität haben und die Orts namenlisten nur ein beliebiger Katalog von Toponymen sind.

5 K. A. Kitchen, On the Reliability of the Old Testament, Cam bridge 2003, 33 . 
Im übrigen ist es bemerkenswert, dass gerade die Triumphdarstellung Schoschenks I. vergleichsweise originell ist, und zwar nicht nur die Ortsnamenliste; auch die Rede Amuns enthält neue und ungewöhnliche Elemente. Zwar sind eine Reihe von Phrasen darin erkennbar älteren Vorbildern entnommen (s. o.), aber der kurze Abschnitt über die Bautätigkeit Schoschenks (Z.16) ist ein Unikum in einer solchen Rede, wie schon Kitchen richtig festgestellt hat (Poetry of Ancient Egypt, 440). Ebenso ungewöhnlich ist es, dass Amun den König dafür lobt, Bauten begonnen zu haben, aber gerade das paßt (gegen Quack, Or 69, 2000, 432) sehr gut zu dem (wenigen), was wir von der Bautätigkeit Schoschenks I. wissen: die Arbeiten sowohl am Tempel von El-Hibeh als auch am ersten Hof von Karnak (dessen Dekoration letztlich unvollendet geblieben ist) sind von seinem Nachfolger Osorkon I. weitergeführt worden. Auch das "Millionenjahrhaus" für Amun, was immer damit gemeint sein mag, wird offenbar auch noch in einer anderen Quelle erwähnt, ${ }^{6}$ es ist kein Hinweis darauf, dass Schoschenk einfach eine ältere Vorlage abschreiben ließ (Quack, loc. cit.) ${ }^{7}$, ganz im Gegenteil. Der Text zeigt sehr schön, wie man in dieser Zeit unter Verwendung älterer Vorbilder eine durchaus eigenständige Komposition anstrebte.

Sinn und Zweck des Feldzugs Schoschenks I. gehen weder aus der ägyptischen Darstellung noch aus dem biblischen Text klar hervor. Ob er seinen früheren Schützling Jeroboam gegen Juda unterstützen wollte, wie Wilson vorschlägt, ob er sich im Gegenteil gegen einen unbotmäßig gewordenen Vasallen (eben Jeroboam) wandte, wie andere gemeint haben, ob es ein reiner Raubzug war oder ob eine nach der Teilung von Salomos Reich günstige Gelegenheit zur Schwächung eines aufstrebenden Nachbarn ausgenützt werden sollte: bis auf weiteres ist es wohl nicht zu entscheiden.

Wilsons Behandlung einer der seltenen Fälle, wo man ein historisches Ereignis in ägyptischen und biblischen Quellen dokumentiert findet, ist insgesamt gut gelungen. Kleinere Versehen beim Umgang mit ägyptischen Texten und Lücken in der herangezogenen Literatur sind bei einem letztlich Fachfremden verzeihlich. Wichtiger ist,

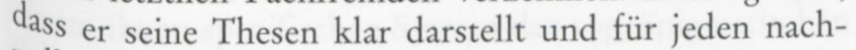
vollziehbar begründet. Dass selbst dann manch einer anderer Meinung sein wird, ist in der Wissenschaft eher die Regel als die Ausnahme.

Vgl. Vernus, BIFAO 75, 1975, 13-14, Z. 6-7; M. Ullmann, König für die Ewigkeit - Die Häuser der Millionen von Jahren, ÄUAT $51,2002,564-75$.

${ }^{7} \mathrm{Zu}$ der von ihm außerdem angeführten Erwähnung des Landes Mitanni s. M. Görg, GM 207, 2005, 5-6. 